\title{
Saprophytic invasion of infarcted pulmonary tissue by Aspergillus species
}

\author{
DR BUCHANAN, D LAMB \\ From the Chest Unit, City Hospital, Edinburgh and the University Department of Pathology, Edinburgh
}

ABSTRACT Saprophytic invasion of infarcted pulmonary tissue has received only passing reference in published reports. ${ }^{1-4}$ Five patients with colonisation of pulmonary infarcts by aspergillus are described, with discussion of the clinical and histopathological features. The characteristic features were widespread invasion of dead tissue by fungal hyphae, in three cases with liquefaction resulting in the production of a cavitated lesion. There was no evidence that the vascular thrombosis and the consequent infarction present in four cases were caused by the fungus. In two cases the lesions were resected as possible peripheral cavitated tumours, and in two they were incidental findings at necropsy in patients who had had carcinoma and received radiotherapy. In the fifth case the clinical diagnosis was pneumococcal pneumonia but the possibility of primary aspergillus pneumonia was raised.

Several species of aspergillus have been identified as pathogens in man, Aspergillus fumigatus being the most frequent. The syndromes described in association with this organism are: as an allergen in bronchial asthma, bronchopulmonary aspergillosis and bronchocentric granulomatosis, extrinsic allergic alveolitis, aspergilloma, and invasive or necrotising aspergillosis. ${ }^{45}$ In addition, $A$ fumigatus may be found as a commensal in sputum. We now suggest that another category should be included-saprophytic invasion of infarcted lung tissue in which there is infiltration of the infarct by fungal hyphae extending to the margins of viable tissue. It is important to differentiate this condition from primary aspergillus pneumonia, a lesion that necessitates antifungal treatment.

\section{Case reports}

CASE 1

A 63-year-old diabetic, who smoked 20 cigarettes a day, had been ill six weeks before admission to hospital with a cough productive of purulent sputum, pleuritic chest pain, and generalised malaise. Chest radiography on admission (fig 1) showed a cavitated peripheral opacity in the left mid zone, suggestive of a peripheral carcinoma. FEV, was 1.6 litres (predicted 2.0 litres), FVC 2.8 litres (the predicted

Address for reprint requests: Dr D Lamb, Department of Pathology, University of Edinburgh, Edinburgh EH3 9YW. value), and $\mathrm{FEV}_{1} / \mathrm{FVC} 57 \%$ (predicted $70 \%$ ). Repeated samples of sputum gave negative results on cytological examination for malignant cells. The findings at fibreoptic bronchoscopy and the bronchial washings were normal. The Mantoux test $1: 10000$ (OT) produced $15 \mathrm{~mm}$ of induration. The patient was started on antituberculosis chemotherapy but there was no radiological improvement. No tissue was obtained from transthoracic percutaneous needle biopsy and he was referred for thoracotomy.

At operation there was a $4-\mathrm{cm}$ nodule in the left upper lobe, adherent to the overlying parietal pleura. In the posterior segment of the resected specimen there was an area of scarring about $3 \mathrm{~cm}$ in diameter containing several small multiloculated central cavities. The margins of the cavities consisted of inflamed granulation tissue and fibrosis with an appreciable inflammatory cellular infiltration, both acute and chronic.

The centre of the cavities contained necrotic lung tissue showing widespread penetration by masses of fungal hyphae, with the septate, branched morphological structure of aspergillus. Adjacent arteries showed recanalised thrombus, but a cross-section of the unaffected subsegmental bronchus draining into the area contained several small aggregates of fungal hyphae.

The appearances were those of an organising true infarct of the lung with saprophytic invasion by the fungus. 


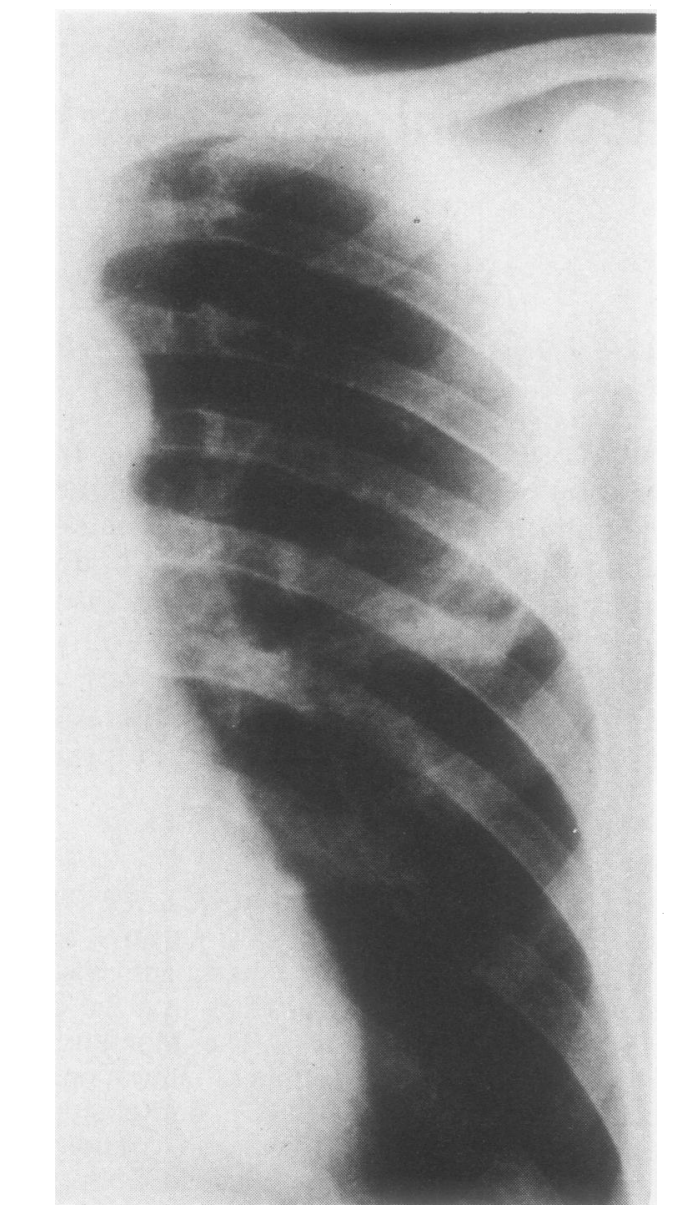

Fig 1 Patient 1: Chest radiograph showing a peripheral cavitated opacity in the left mid zone.

\section{CASE 2}

A 64-year-old retired miner, a heavy smoker of 60 cigarettes a day with a long history of chronic bronchitis, was admitted with a two-week history of cough and purulent sputum. Chest radiography showed the changes of consolidation throughout the left upper zone (fig 2). No pathogenic bacteria or malignant cells were identified from sputum. After two weeks of treatment with ampicillin there was partial radiological clearing but his chest radiograph still showed a rounded opacity lying in the apical segment of the left lower lobe. FEV , was 1.9 litres and FVC 3.1 litres. Liver scanning, barium swallow, and cytological examination of sputum gave normal results.

The patient was referred for thoracotomy and underwent left lower lobectomy for presumed bron- chogenic carcinoma. At operation there was an illdefined mass at the apex of the left lower lobe, adherent to the chest wall posteriorly, and a left lower lobectomy was performed. There was a large area of subpleural scarring in the apical segment of the left lower lobe. The lung had been incised at that point and contained a cavity filled with soft, cheesy material. Thrombus was identified, occluding vessels adjacent to the cavity. Microscopically, sections confirmed an area of scarring in the apical segment of the left lower lobe associated with a cavity. The inner aspect of the cavity consisted of granulation tissue extending out to the pleura. The cavity contained infarcted lung that had been colonised by $A$ fumigatus. There was no evidence of tuberculosis or tumour associated with the lesion. The blood vessels around the lesion were thrombosed, some showing recanalisation and others an associated inflammatory reaction.

\section{CASE 3}

A 54-year-old man, who smoked over 40 cigarettes a day and had a four-month history of hoarseness, breathlessness, and dysphagia with a weight loss of over $6 \mathrm{~kg}$, was noted to have a hoarse voice, clubbing of the fingers, and small lymph nodes in the neck on examination. Radiological investigations showed an abnormal left hilum with slight indentation of the anterior wall of the oesophagus caused by enlarged mediastinal glands. At bronchoscopy the left vocal cord was paralysed and the left upper-lobe bronchus was seen to be narrowed because of extrinsic pressure from enlarged glands. No biopsy specimen was taken. He received a course of radiotherapy with little radiological response.

Three years later the patient was readmitted with a six-week history of increasing breathlessness and signs of a pleural effusion. He deteriorated and died shortly afterwards. The left pleural cavity was obliterated by white tumour tissue encasing the entire surface of the lung. There was extensive tumour in the pericardial cavity enveloping the pulmonary vessels with partial obstruction. On section of the left lung a tumour was found arising from the left upper-lobe bronchus at its origin and infiltrating circumferentially from the hilum, surrounding the main bronchus, major pulmonary veins, and arteries and extending peripherally around the smaller bronchi. The main tumour mass measured about $10 \mathrm{~cm}$ in maximum diameter and extended out to the pleural aspect, which was covered by tumour tissue. Distal to the tumour there was patchy consolidation. Histologically the tumour consisted of islands of moderately well-differentiated mucin-secreting adenocarcinoma surrounded by dense reactive fibrous tissue. In addition, there was extensive nec- 


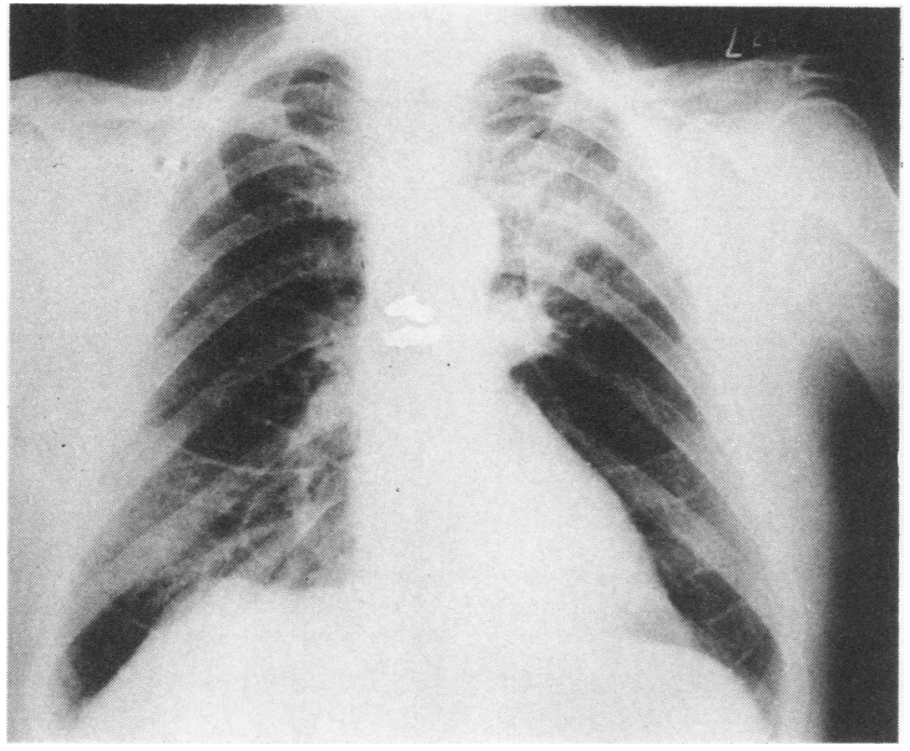

Fig 2 Patient 2: Chest radiograph on admission showing the changes of consolidation throughout the left upper zone.

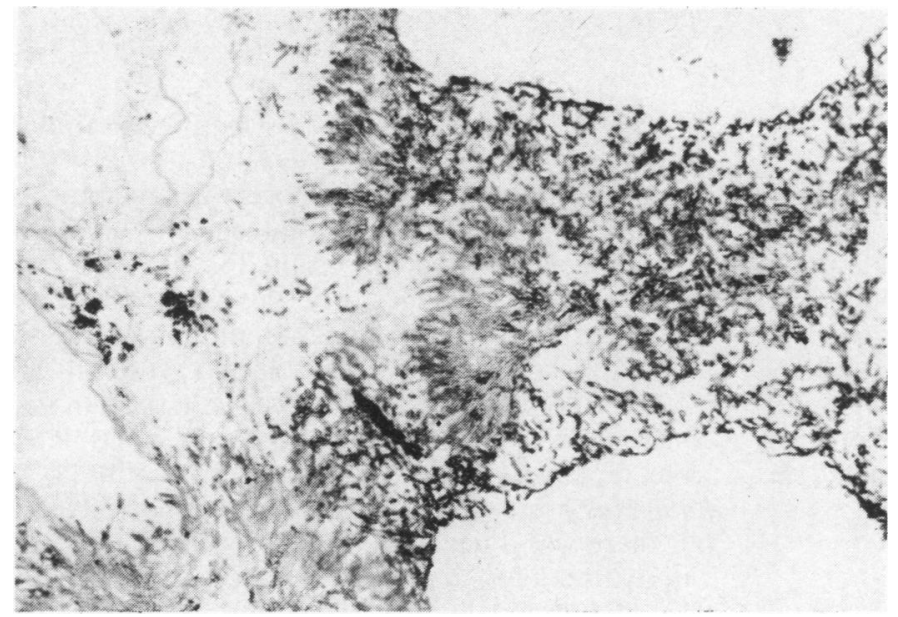

Fig 3 Patient 3: Infarcted lung tissue with invasion by colonies of Aspergillus fumigatus (methenamine-silver preparation, original magnification $\times 125$ ).

rosis of the lung with thrombosis of larger branches of the pulmonary artery, probably as a consequence of radiotherapy. The arteries showed old organised thrombus in their lumina and in some areas there was recanalisation. There was saprophytic invasion of the infarcted tissue by fungal hyphae with actively growing aspergillus eroding through dead cartilage and necrotic bronchial walls (fig 3 ). There were no metastatic deposits either in the liver or in lymph nodes and sections of the other organs showed no important histopathological features.
CASE 4

A 75-year-old man with chronic bronchitis, who smoked 10 cigarettes a day, developed a cavitating lesion in the right mid zone. As he was unfit for operation, he underwent a course of radiotherapy in view of the presumed diagnosis of bronchogenic carcinoma. He was admitted a year later with an acute respiratory infection and subsequently died.

At necropsy some fine fibrous adhesions were noted in the right pleural cavity overlying the lateral surface of the right middle lobe, which contained an 


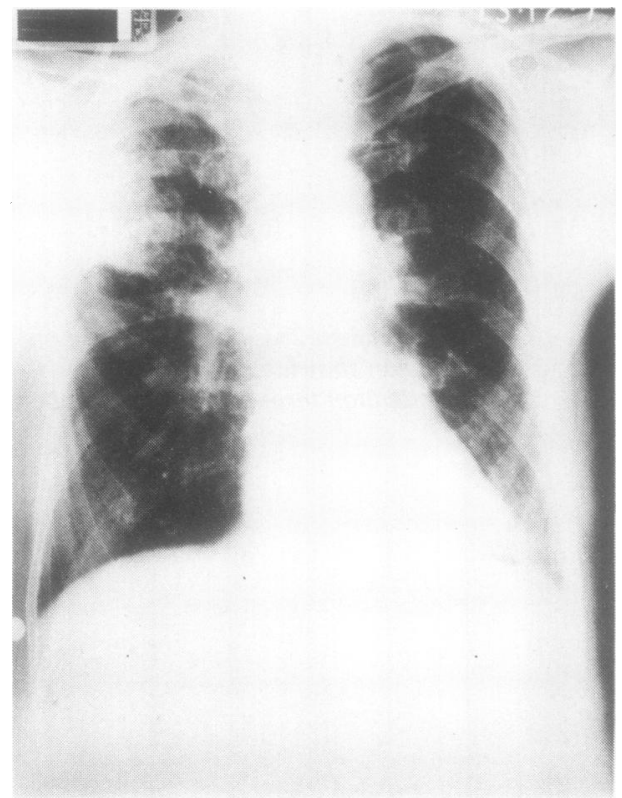

Fig 4 Patient 5: Chest radiograph on admission with consolidation shadowing in the right upper zone.

irregular necrotic tumour mass measuring $5.5 \mathrm{~cm}$ in maximum diameter. Microscopically, the tumour had the histological structure of a moderately differentiated squamous carcinoma. The upper lobe contained an area of infarction measuring $5 \mathrm{~cm}$ in maximum diameter with early cavitation. Microscopically there was extensive invasion of infarcted tissue by fungal hyphae with the morphological characteristics of aspergillus. Vessels proximal to the infarct showed organising thrombus.

\section{CASE 5}

A 78-year-old railway driver had been ill for three weeks before being admitted to hospital with a productive cough, right-sided pleuritic chest pain, and increased exertional dyspnoea. He had received a year's course of antituberculosis chemotherapy several years previously, although the diagnosis had not been confirmed bacteriologically. His chest film at that time had shown minor fibrotic changes with calcification at both apices. Thereafter he had been seen regularly for some years but was later discharged from routine follow-up.

On admission he looked ill, was dyspnoeic at rest, and had a fever of $38^{\circ} \mathrm{C}$. Scattered crepitations were audible and the chest radiograph showed inflammatory shadowing in the right upper zone (fig 4). The sputum was purulent and yielded a growth

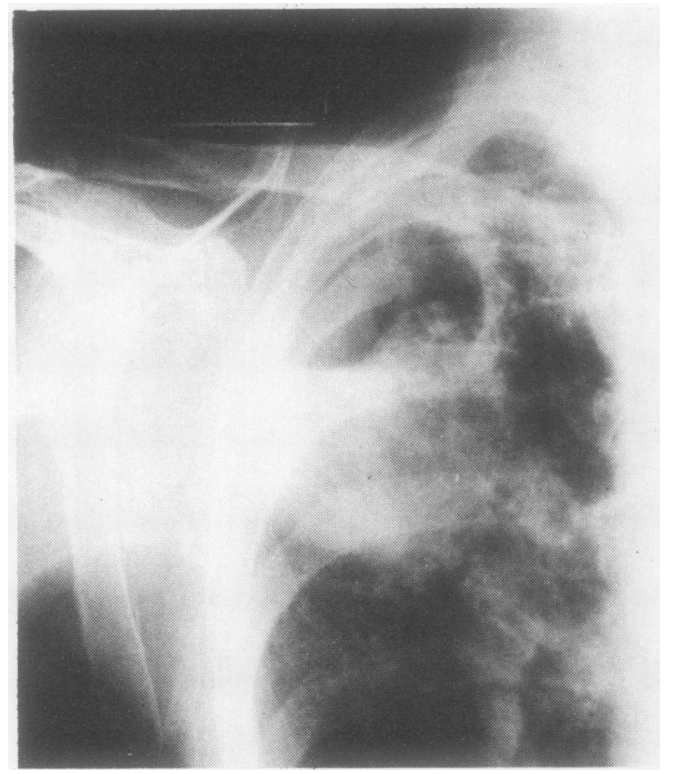

Fig 5 Patient 5:Chest radiograph shortly before death showing well-marked cavitation in the right upper zone.

of type III pneumococci. The peripheral blood showed a neutrophil leucocytosis of $13.0 \times 10^{9} / 1$. The presumptive clinical diagnosis was pneumococcal pneumonia and he was treated with parenteral benzyl penicillin and ampicillin for 12 days. His condition began to improve but his chest radiograph showed progressive consolidation in the right upper lobe with the development of early cavitation. He produced copious quantities of purulent sputum that contained eosinophils, and $A$ fumigatus was isolated from repeated specimens. Serum precipitins to $A$ fumigatus were identified in the peripheral blood, but there was a negative response to skin prick test with extract of $A$ fumigatus. As the patient's condition had gradually improved and his fever had settled the antibiotics were discontinued, but his chest radiograph showed well-marked cavitation in the $\frac{D}{0}$ right upper zone (fig 5). A fumigatus continued to be isolated from sputum in large numbers and aspergil- $N$ lus precipitins were still present in his serum.

The possiblity of secondary necrotising aspergillus infection was considered, but in view of his clinical $\omega$ wellbeing it was decided to withold antifungal treatment. Three days later he died suddenly.

At necropsy the main findings were confined to $\mathbb{D}$ the lung. The right upper lobe contained an extensive infarct measuring $10 \times 8 \times 6 \mathrm{~cm}$ extending out to the pleural aspect and involving the whole of the apical segment. The bulk of the infarct had separated to form a large central slough leaving a cavity 


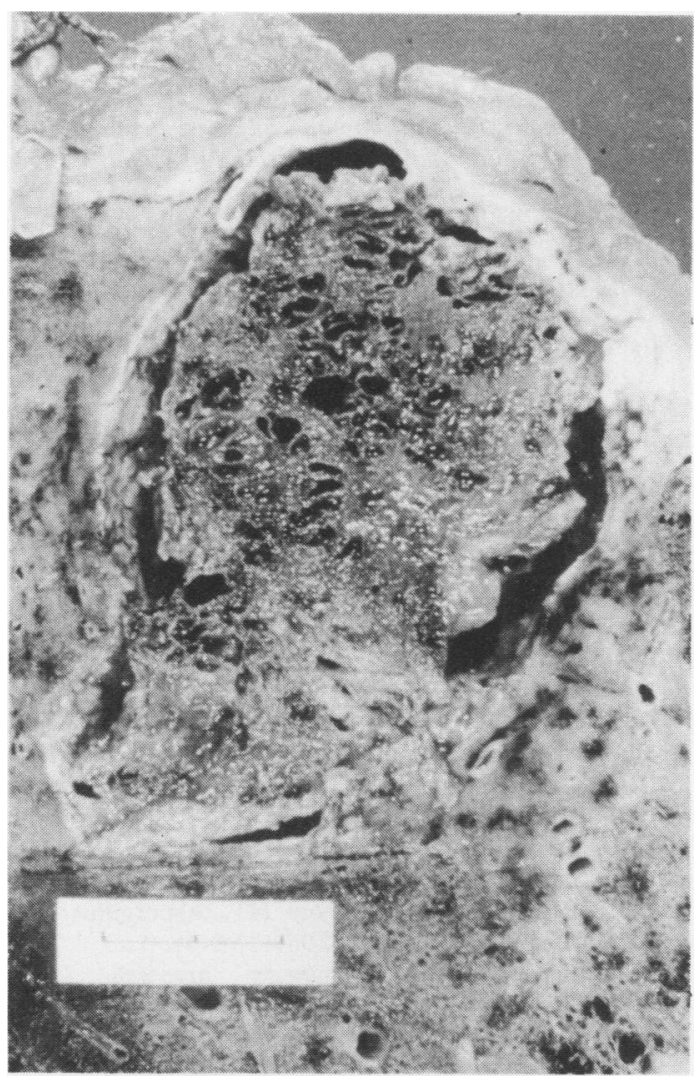

Fig 6 Patient 5: Section of the right upper lobe at necropsy showing an infarct, with separation and shrinkage around its margins producing cavitation (scale indicates $2 \mathrm{~cm}$ ).

(fig 6). Microscopically, both the corrugated membrane lining the cavity and the central slough consisted of fibrin, debris, and a felted network of fungal hyphae (fig 7). There was no evidence of old cavity formation in the upper lobe. The upper-lobe artery to this area showed laminated in-situ thrombus, but no thrombus was seen in the major pulmonary vessels. Surrounding the infarct there was acute bronchopneumonic consolidation, with evidence of older changes of organisation in the posterior segment of the upper lobe. No specific cause was established for the terminal episode, which appeared to be unrelated to the fungal infected infarct and was considered to be due to a sudden cardiac arrhythmia.

\section{Discussion}

Although it is not recognised as one of the main classical patterns of lung disease caused by aspergillus, ${ }^{56}$ saprophytic invasion of infarcted pulmonary tissue, of which we describe five examples, may be more common than is appreciated. The characteristic features in all five cases were widespread penetration of dead tissue by proliferating fungal hyphae with, in three cases, liquefaction and cavitation. This differs from the necrotising form of opportunistic invasive aspergillosis in that the tissue death came first. In none of the cases did the appearances suggest secondary necrosis of tissue as a consequence of fungal growth originating primarily in airways, as is seen in opportunistic invasive aspergillosis. ${ }^{34}$

In four cases there was evidence of old thromboembolism in vessels proximal to the areas of infarction and well away from the saprophytic fungal invasion of dead tissue. The fact that aspergillus can saprophytically colonise infarcted tissue has been known since $1856 .^{89}$ In most cases colonisation of infarcted lung tissue by aspergillus is an incidental pathological finding, with no evidence of true parasitic invasion. ${ }^{2}$ The fungus can also saprophytically colonise and multiply on the surface of walls of preformed lung cavities caused by tuberculosis, bron-

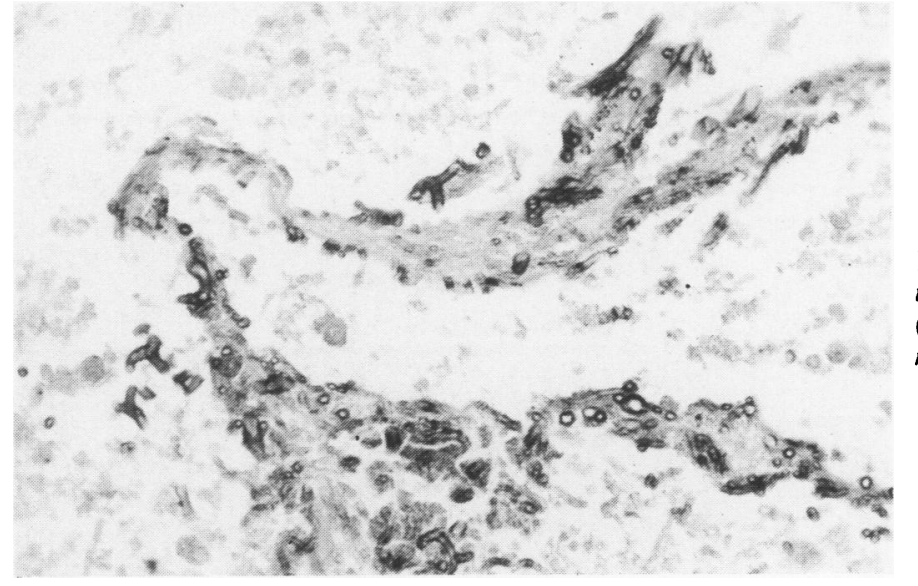

Fig 7 Patient 5: Infarcted lung tissue invaded by colonies of Aspergillus fumigatus (methenamine-silver preparation, original magnification $\times 320$ ). 
chiectasis, congenital cystic disease, emphysema, suppuration, and neoplasia and even cavities resulting from other fungal infections, notably histoplasmosis, without actual penetration of adjacent lung tissue. ${ }^{34}$ Possibly progressive excavation of infarcted tissue by the fungus might lead to the formation of an aspergilloma or mycetoma in the cavity so formed, giving rise to the development of an intracavitary fungal-ball colony. We have not found a record of this in the published reports.

Primary aspergillus pneumonia is stated to occur in the absence of the usual predisposing causes. ${ }^{3410}$ In some instances the diagnosis has been claimed simply because aspergillus has been found in sputum in the presence of pneumonia. " This alone gives insufficient grounds for such a diagnosis; and possibly saprophytic invasion of infarcted pulmonary tissue constitutes the basis for some reported cases of fungal pneumonia in the non-compromised host, ${ }^{12}$ which-if it exists-is a relatively infrequent occurrence.

We are indebted to Dr NW Horne, City Hospital, Edinburgh, for his invaluable help and assistance and to Dr GJR McHardy, Mr PR Walbaum, and the late Mr RJM McCormack for allowing us to study patients under their care. We are grateful to Miss $\mathbf{J}$ Holywell for typing the manuscript.

\section{References}

' Symmers WStC. In: de Haller R, Suter F. Aspergillosis and farmer's lung in man and animals. Proceedings of the fourth international symposium, October 1971. Bern: Huber, 1974:75-87.

${ }^{2}$ Symmers WStC. Histopathologic aspects of the pathogenesis of some opportunistic fungal infections, as exemplified in the pathology of aspergillosis and phycometoses. Lab Invest 1962;2:1073-90.

${ }^{3}$ Spencer H. In: Pathology of the lung. 3rd ed. Vol 1. Oxford: Pergamon Press, 1977:275-82.

${ }^{4}$ Payling Wright G, Heard BE (revised Heard BE). Systemic pathology. 2nd ed. Vol 1. Edinburgh: Churchill Livingstone, 1976:349-51.

${ }^{5}$ Turner-Warwick M. Aspergillus fumigatus and lung disease. Postgrad Med J 1979;55:642-4.

- Turner-Warwick M. Immunology of the lung. Curr Top Immunol 1978;10.

7 Young RC, Bennett JE, Vogel CL, Carbone PP, Devita NT. Aspergillosis: the spectrum of the disease in 98 patients. Medicine 1970;49:147-73.

${ }^{8}$ Virchow VR. Beiträge zur Lehre von den beim Menschen vorkommended pflanzlichen Parasiten. Virchow; Archiv für Pathologische Anatomie und Physiologie 1856;9:557-93.

${ }^{9}$ Hinson KFW. Moon AJ, Plummer NS. Bronchopulmonary aspergillosis. Thorax 1952;7:317-33.

${ }^{10}$ Young RC, Vogel CL, Devita NT. Aspergillus lobar pneumonia. JAMA 1963;208:1156-62.

"Delikat E. Acute pulmonary mycosis. Lancet 1945;ii:370-1.

12 Kennedy WPU, Malone DN, Blyth W. Necrotising pulmonary aspergillosis. Thorax 1970;25:691-701. 